\title{
Performance analysis of optical extra-WBAN links based on realistic user mobility modeling
}

\author{
Oussama Haddad $\odot$, a Mohammad-Ali Khalighi $\odot, \stackrel{\text { a,* }}{,}$ \\ and Stanislav Zvanovec $\odot^{b}$ \\ ${ }^{a}$ Aix-Marseille University, CNRS, Centrale Marseille, Institut Fresnel, \\ Marseille, France \\ ${ }^{\mathrm{b}}$ Czech Technical University in Prague, Department of Electromagnetic Field, \\ Faculty of Electrical Engineering, Prague, Czech Republic
}

\begin{abstract}
We address the features channel characterization and performance evaluation for wireless body-area networks (WBANs) in medical applications during a walk scenario using optical wireless transmission. More specifically, we focus on optical extra-WBAN uplink communication between a central coordinator node $(\mathrm{CN})$ placed on the patient's body and an access point (AP) in a typical hospital room. To characterize the optical wireless channel, we use a Monte Carlo ray tracing-based method and take into account the effects of body shadowing and mobility based on realistic models, in contrast to the previous simplistic models considered in the literature. Using this approach, we derive the dynamic behavior of the channel DC gain for different configurations of the CNs and APs. Furthermore, based on the obtained results, we develop a statistical channel model based on kernel density estimation, which we use to investigate the impact of $\mathrm{CN}$ and AP placement on the communication link parameters. Also, based on the outage probability criterion, we discuss the link performance and further analyze the improvement in performance achieved through spatial diversity, i.e., by using multiple APs in the room, for different photodetector types, under different background noise conditions. The presented results show that $\mathrm{CN}$ placement and user's local and global mobility significantly impact the performance of extra-WBAN links, which can nevertheless be reduced using spatial diversity. Finally, the presented performance analysis shows that a single AP equipped with an avalanche photodiode photodetector allows an acceptable link performance for low-to-moderate background noise conditions, whereas multiple APs equipped with PIN photodetectors should be used in the case of moderate-to-strong background noise. () The Authors. Published by SPIE under a Creative Commons Attribution 4.0 International License. Distribution or reproduction of this work in whole or in part requires full attribution of the original publication, including its DOI. [DOI: 10.1117/1 .OE.61.2.026113]
\end{abstract}

Keywords: wireless body-area networks; medical wireless body-area networks; extra-wireless body-area network links; optical wireless communications; optical channel characterization; user mobility; Monte Carlo ray tracing; outage probability analysis.

Paper 20210582 received Jun. 2, 2021; accepted for publication Jan. 27, 2022; published online Feb. 26, 2022.

\section{Introduction}

E-health solutions are attracting increasing attention in many application scenarios as efficient systems for improving the quality of life and reducing health-care expenditures. ${ }^{1}$ This popularity is driven by the advances made in different fields of engineering. The very large-scale integration of sensors and actuators in millimeter size chips ${ }^{2,3}$ and the development of wireless communication technologies ${ }^{4}$ allow relatively low-cost practical implementations of these solutions. Typical application examples include real-time remote monitoring of patients and elderly people in health-care centers or at home. Medical wireless body-area networks (WBANs) can be considered as the main building block of such systems by enabling the acquisition and transmission of timely data from several medical sensors. The most common architecture of WBANs is a star topology that connects these sensors to a coordinator node $(\mathrm{CN})$, placed on the patient's body.

*Address all correspondence to Mohammad-Ali Khalighi, Ali.Khalighi@fresnel.fr 
The $\mathrm{CN}$ then transmits the collected data to a mobile device or a fixed access point (AP) through what is called an extra-WBAN link. ${ }^{5}$

Most previous works on extra-WBAN links have been based on the radio frequency (RF) technology, primarily using the unlicensed ISM (industrial, scientific, and medical) frequency bands. For instance, in Refs. 6 and 7, analytical models were proposed for describing body movements and postures in WBAN scenarios. In Ref. 8, a soldier-to-soldier communication link was characterized using an avatar derived from three-dimensional (3D) animation. Also, a motion capture-based model was used in Refs. 9 and 10 to study the extra-WBAN channel. However, RF-based solutions are subject to electromagnetic interference with the other existing networks that use the ISM band, in addition to data security issues that may arise. Also, the transmit power level is restricted to satisfy the specific absorption rate to avoid damaging the patients' tissues. ${ }^{11}$

These considerations have motivated the investigation of optical wireless communications (OWC) as an alternative or complementary solution to RF data transmission for medical WBANs. The main advantages of OWC are their immunity to external interference, their inherent security due to the confinement of light in indoor environments, and the negligible impact of multipath (small scale) fading. ${ }^{12}$

Note that the optical and RF WBAN channels are fundamentally different in terms of smallscale fading and sensitivity to shadowing. A few studies have considered OWC-based WBANs in the infrared band. For instance, Ref. 13 studied the optical intra-WBAN channel using a simplified model for the patient's body and mobility. In previous works, ${ }^{14,15}$ we considered intraWBAN channel characterization and performance evaluation using more sophisticated body mobility models. However, the fundamental differences between intra- and extra-WBAN links, in particular, in terms of shadowing and link blockage, necessitate a dedicated study for the latter case.

To study the OWC-based extra-WBAN links, a Monte Carlo ray tracing (MCRT)-based simulation tool is used in Ref. 16 to study a physical activity monitoring system for a moving patient while neglecting the effect of the patient's body. The authors in Ref. 17 studied downlink extraWBAN transmission while neglecting local body movements and assuming that the receiver (Rx), i.e., the $\mathrm{CN}$, is at a short distance from the body. In Refs. 18 and 19, user mobility was taken into account based on uniform-distributed random user positions and random orientations of sources and detectors. However, Ref. 18 neglected the body model, and Ref. 19 considered a 3D rectangular shape model, without any consideration for local mobility, and hence body parts' shadowing. Neglecting the effect of mobility, Ref. 20 proposed a site-specific ray tracing approach that considers the skin tissue for the channel modeling of WBAN communication links in hospital scenarios. Finally, neglecting the user's body modeling, Ref. 21 studied the performance of optical code-division multiple access (O-CDMA) for uplink extra-WBANs considering the orientation-based random waypoint (RWP) model for user mobility. A preliminary experimental study on the use of O-CDMA for extra-WBANs was further presented in Ref. 22. For a more comprehensive survey on channel modeling for optical WBANs, the reader is referred to Ref. 20. Note that, in addition to the simplified body and mobility models used, the considered models in the above-mentioned works do not take into consideration the notion of walk trajectories and, hence, neglect the correlation that exists between consecutive sequences of the movement.

This work investigates channel modeling and performance of OWC-based extra-WBANs in the uplink. In contrast to the previous works on the topic, this study is based on more accurate and realistic channel models with an acceptable level of details, in particular, concerning the body's 3D shape and user mobility. More precisely, a dynamic mobility model based on a 3D animation of a walk cycle is proposed to model body parts movement. Also, a modified RWP model is used for the trajectories taken by the patient within the room. This accuracy in modeling the body and its movements is a key requirement for accurate channel characterization since the change of body postures can cause variable shadowing that can significantly affect the link availability. Also, the time correlation between consecutive sequences of the walk trajectory needs to be taken into account in the system design. Our study is primarily based on the calculation of the channel impulse response (CIR) and its main related parameters. The link performance is quantified in terms of the outage probability considering the on-off keying (OOK) modulation. We further investigate the performance improvement achieved by using 
multiple APs in the room. Two cases of PIN and avalanche photodiode (APD)-based Rxs are considered with different background noise conditions.

The remainder of the paper is organized as follows. Section 2 presents the considered simulation approach and parameter specification. Then, numerical results are provided in Sec. 3 to study the channel DC gain for different configurations. The link outage probability is evaluated in Sec. 4, where the performance gain offered by using multiple APs is also studied. Section 5 concludes the paper.

\section{Simulation Approach}

This section describes our approach to optical channel characterization and performance analysis.

\subsection{Channel Characterization}

To estimate the channel CIR, we perform MCRT simulations using Opticstudio software from Zemax, ${ }^{23}$ which can model complex 3D environments. The interest in using comprehensively sophisticated and extremely-time-consuming ray tracing simulations with OpticStudio is that the obtained results are close enough to reality as it was already demonstrated in Ref. 24. For an OWC link, the CIR $h(t)$ is calculated as follows ${ }^{14}$ :

$$
h(t)=\frac{1}{N_{\text {ray }}} \sum_{i=1}^{N_{\text {ray }}} P_{\text {ri }} \delta\left(t-\eta_{i}\right),
$$

where $N_{\text {ray }}$ is the number of rays that hit the detector, $P_{\mathrm{ri}}$ is the received power of the $i$ 'th ray, and

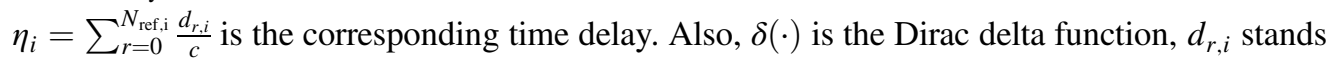
for the optical path length between consecutive reflection surfaces hit by the ray, $N_{\text {ref, } i}$ is the reflection order of the $i$ 'th ray, and $c$ denotes the speed of light. Note that $h(t)$ accounts for both the line-of-sight (LOS) and diffuse links that result from first- and higher-order reflections. A more detailed description of CIR calculation using Opticstudio can be found in Ref. 14.

Based on the calculated CIR, the main metric that determines the signal-to-noise ratio at the $\mathrm{Rx}$ is the channel DC gain, defined as $H_{0}=\int h(t) \mathrm{d} t .{ }^{12,25}$ Other relevant channel metrics include the delay spread $\tau$ and the coherence time $T_{c}$. The parameter $\tau$ determines channel frequency selectivity and the need to channel equalization, whereas $T_{c}$ describes channel time variations based on which are fixed the frame length and training sequences [used for channel estimation and adaptive power control at the transmitter (Tx)]. ${ }^{26}$ In Ref. 27, we showed that the optical extra-WBAN channels can be considered as frequency non-selective up to a data rate of $10 \mathrm{Mbps}$. Also, $T_{c}$ varies between 0.9 and $4.2 \mathrm{~s}$, which indicates slow channel time variations. Readers are referred to Ref. 27 for detailed numerical results. Consequently, in the sequel, we only focus on the channel DC gain as the main channel metric. Compared to Ref. 27, in this paper, we propose a more detailed investigation and interpretation of channel characterization with more accurate statistical models based on additional walk trajectories. Furthermore, we investigate the link performance for different Rx types, taking into account different noise sources. Also, we consider new configurations with different signal combination strategies in the case of using multiple APs.

\subsection{User Mobility Modeling}

To study the effect of user mobility, we take into account both the changes in the position of the user inside the room and the movement of the body parts, i.e., the arms, legs, etc., that we will refer to as global and local mobility, respectively.

For local mobility, we model the body by a 3D mesh ${ }^{28}$ composed of 902 vertices, which are connected to form 1800 triangular faces. This mesh provides an acceptable level of detail to take into account shadowing effects from the body segments. The body movements are modeled 


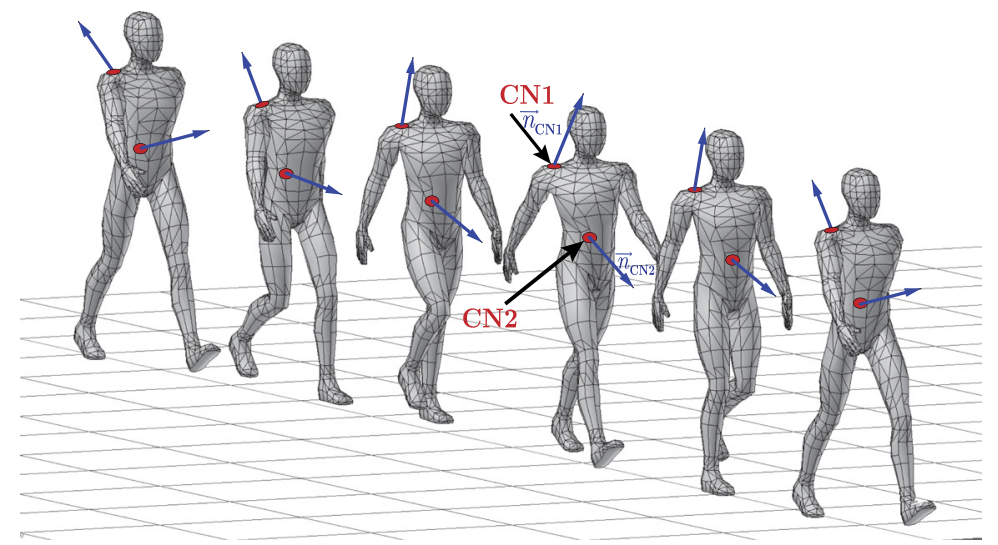

(a)

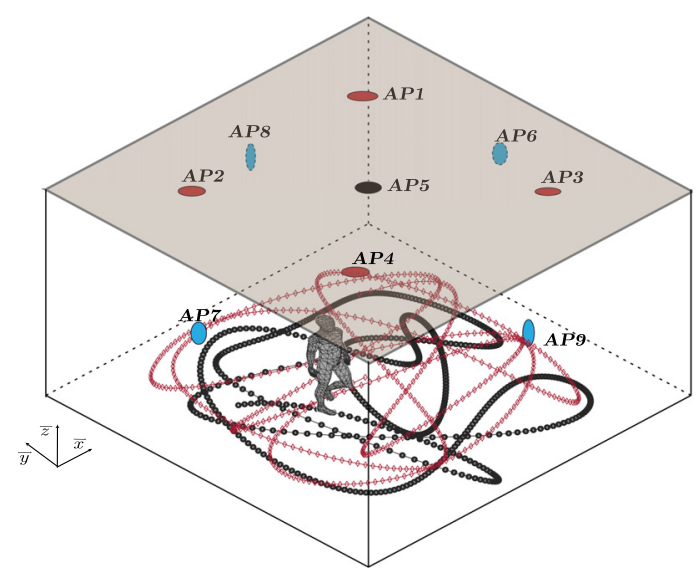

(b)

Fig. 1 (a) Illustration of the mobility configuration with the considered placements for the CNs. (b) Considered placements of APs and the simulated walk trajectory (AP1 to AP5 are placed on the ceiling and AP6 to AP9 on the walls).

using a 3D walk cycle animation generated by the Blender software. ${ }^{29}$ The walk cycle consists of $N_{f}=27$ frames representing body configurations at different instants of the walk. Guidelines for creating the walk cycle animation are available in Ref. 30. Figure 1(a) illustrates a few frames of the walk cycle.

For global mobility, a modified RWP mobility model ${ }^{14}$ is used, where, instead of linear interpolation between the waypoints, ${ }^{31}$ we use cubic splines to generate a curved trajectory. ${ }^{32}$ This allows to consider a smooth path without sharp rotations, which is more realistic. The synchronization between the local and global mobility is done by calculating the optimal time step $\Delta t$ for which we change the consecutive walk cycle frames, and it is given by

$$
\Delta t=\frac{1}{N_{f}} \frac{d_{\text {cycle }}}{v_{\text {user }}},
$$

where $N_{f}$ denotes the number of frames of the walk cycle, $d_{\text {cycle }}$ is the distance traveled by the user during a walk cycle, and $v_{\text {user }}$ is the user speed during that time. Considering a reasonable $d_{\text {cycle }}$ of $2 \mathrm{~m}$ and a walk speed of $0.45 \mathrm{~m} / \mathrm{s},{ }^{33,34}$ we have $\Delta t \approx 0.17 \mathrm{~s}$.

As suggested in Refs. 13 and 19, two positions are considered for the CN, i.e., on the shoulder and on the waist that we will refer to as $\mathrm{CN} 1$ and $\mathrm{CN} 2$, respectively. Both $\mathrm{CN} 1$ and $\mathrm{CN} 2$ are oriented perpendicular to their respective triangular faces [see Fig. 1(a)]. Note that as the body moves, the configuration of its triangular faces changes, thus, the positions and orientations of the CNs, placed on these faces will change accordingly. In fact, the reliability of the link 
between the $\mathrm{CN}$ and the $\mathrm{AP}$ may depend on the $\mathrm{CN}$ placement. For example, the link corresponding to $\mathrm{CN} 1$ is less sensitive to shadowing from body parts than $\mathrm{CN} 2$, whereas the link corresponding to $\mathrm{CN} 2$ is more subject to diffuse links through first-order reflections.

We consider different placements for the APs on the ceiling and the walls, as shown in Fig. 1(b) together with the two generated trajectories shown in black and red using the modified RWP model. As can be seen, these trajectories cover most of the room area. This allows the derivation of accurately enough mobility statistics, as we will see later in Sec. 4. Also, AP1 to AP5 are symmetrically placed on the ceiling and point downward, whereas AP6 to AP9 are placed on the walls perpendicularly at half-width and 2-m height.

\subsection{Signal Transmission Formulation}

Justified by its implementation simplicity, intensity modulation with direct detection based on uncoded non-return to zero (NRZ) OOK modulation is considered. The received signal at the photodetector (PD) output is

$$
r_{n}=\mathcal{R} G P_{k} H_{0}+\mathrm{n}_{n},
$$

where $\mathcal{R}$ and $G$ stand for the PD responsivity and gain, respectively. Also, $P_{k}$ denotes the power of the transmitted symbol, which equals $P_{1}$ for ON symbols or $P_{0}=\alpha P_{1}$ for OFF symbols, with $\alpha$ being the extinction ratio. The average transmit power is $P_{t}=\frac{(1+\alpha) P_{1}}{2}$. Furthermore, in Eq. (3), $\mathrm{n}_{n}$ is the total photocurrent noise that accounts for thermal, background, dark, and shot noises. It is modeled as Gaussian with variance $\sigma_{\text {tot }}^{2}$ and given by

$$
\sigma_{\mathrm{tot}}^{2}=\sigma_{0}^{2}+\sigma_{s, k}^{2},
$$

where $\sigma_{0}^{2}=\sigma_{\mathrm{th}}^{2}+\sigma_{\mathrm{b}}^{2}$ with $\sigma_{\mathrm{th}}^{2}=4 K_{b} T_{e} B / R_{L}$ and $\sigma_{\mathrm{b}}^{2}=2 e G^{2} F B I_{b}$ being the variances of thermal and background noises, respectively. ${ }^{35}$ Here, $e$ is the electron charge, $K_{b}$ is the Boltzman constant, $T_{e}$ the equivalent noise temperature, and $F$ the PD excess noise factor. Also, $B$ denotes the Rx low-pass filter (LPF) bandwidth, typically approximated by half of the data rate $B \approx R_{b} / 2, R_{L}$ is the load resistor of the trans-impedance amplifier (TIA), and $I_{b}$ is the average photocurrent generated by a PIN PD due to the background noise. ${ }^{36}$ Besides, in Eq. (4) the variance of the shot noise corresponding to OFF and ON symbols is denoted by $\sigma_{s, k}^{2}=$ $2 e G F B I_{k}$, with $I_{k}=\mathcal{R} G P_{k} H_{0}$ being the received current of the corresponding symbol. We reasonably neglect the dark current noise compared with the other noise sources. ${ }^{35}$

\subsubsection{Statistical channel modeling}

To avoid time-consuming MCRT simulations with Opticstudio, we propose a statistical model for the channel DC gain. The model is developed based on the nonparametric kernel density estimation (KDE), ${ }^{37}$ which is applied to the obtained results for the simulated user trajectory (see Sec. 2.2). Note that, in contrast to the classical parametric approach, where the probability density function (PDF) is assumed to be known up to a finite number of parameters, the nonparametric methods assume that the PDF belongs to an infinite collection of functions and, hence, offer more modeling flexibility and are not affected by the choice of an inappropriate parametric model. ${ }^{38,39}$

The KDE model is defined for a set of $N$ realizations $x_{i}$ of a random variable $x$ with the PDF $f_{X}(x)$. The KDE estimate of the PDF is given by ${ }^{40}$

$$
\hat{f}_{X}(x)=\frac{1}{N \rho} \sum_{i=1}^{N} \mathcal{K}\left(\frac{x-x_{i}}{\rho}\right),
$$

where $\mathcal{K}$ is the kernel and $\rho=1.06 \hat{\sigma} N^{-1 / 5}$ is the smoothing parameter. Here, $\hat{\sigma}$ is the estimated standard variation of $\log (x)$ related to the dataset of Eq. $(5) .^{41}$ For the sake of simplicity, a Gaussian kernel is considered here. 


\subsubsection{Bit-error rate calculation}

Using the statistical models obtained in the Sec. 2.3.1, we randomly generate $10^{7}$ values of $H_{0}$ for every CN-AP extra-WBAN link. Then, for each channel realization, we calculate the bit-error rate (BER) for an uncoded NRZ OOK-based link for a given $R_{b}$ and $P_{t}$. Assuming optimal maximum likelihood detection taking into account the signal-dependent noise, we have $\mathrm{e}^{42}$

$$
\mathrm{BER}=\frac{1}{4} \operatorname{erfc}\left(\frac{\gamma_{\mathrm{th}}-\alpha I_{1}}{\sqrt{2\left(\alpha \sigma_{s, 1}^{2}+\sigma_{0}^{2}\right)}}\right)+\frac{1}{4} \operatorname{erfc}\left(\frac{I_{1}-\gamma_{\mathrm{th}}}{\sqrt{2\left(\sigma_{s, 1}^{2}+\sigma_{0}^{2}\right)}}\right),
$$

where $\operatorname{erfc}(x)=\frac{2}{\sqrt{\pi}} \int_{x}^{\infty} \exp \left(-u^{2}\right) \mathrm{d} u$ is the complementary error function and $\gamma_{\mathrm{th}}$ is the optimal detection threshold. Also, $I_{1}$ is the PD output photocurrent for ON symbols.

\subsection{Parameter Specification}

To ensure accurate enough CIR estimation, $N_{\text {rays }}=2.5 \times 10^{7}$ rays are considered for the MCRT simulation with at least three-order reflections for beam propagation. The Blender software is used to create the 3D scene for the simulator, similar to the approach used in Ref. 14. A Python script is then used to load a set of configurations specifying user movements and orientations for $N_{\text {config }}=1103$ sequential snapshots of a walk scenario. We consider two trajectories of 516 and 587 sequential snapshots as explained previously and illustrated in Fig. 1(a), which, overall, give an acceptable coverage of the room area and a detailed enough statistical description of the walk cycles. Note that generating these data has necessitated quite long Opticstudio simulations.

For the sake of simplicity, we consider an empty room, i.e., without any furniture or medical devices, with dimensions $(5 \times 5 \times 3) \mathrm{m}^{3}$ with plaster walls and ceiling, and pinewood floor. The reflectance of materials is modeled by a wavelength-dependent classical Lambertian bidirectional reflectance distribution function. ${ }^{12}$ The reflection properties of the different surfaces are fixed following Ref. 43. Given the low reflectivity of the skin compared to that of the walls, ${ }^{44}$ the user body is assumed to be absorbent. Although cloths' reflectivity can range from 0.02 to $0.75,{ }^{45}$ we choose here the worst-case scenario that maximizes beam shadowing effects.

At the Tx (i.e., CN) side, an LED with wavelength $\lambda_{0}=850 \mathrm{~nm}$ is considered with a bandwidth of $\Delta \lambda=30 \mathrm{~nm}$ and a Lambertian radiation pattern. ${ }^{12}$ To ensure minimum beam blockage, a Lambertian order of $m=1$ is assumed, corresponding to a semi-angle of $60 \mathrm{deg}$. At the Rx (i.e., AP) side, the PD is assumed to have an $1 \mathrm{~cm}^{2}$ active area, without any lens and with a large field-of-view (FOV) of $60 \mathrm{deg}$ (semi-angle). The relatively large PD surface is justified by the relatively low data rates, on the order of $1 \mathrm{Mbps}$ at most, required in medical WBANs. ${ }^{46}$ Two types of a PIN PD and an APD (with $G=50$ and $F=3$ ) are considered in this study. Table 1 summarizes the main system parameters.

Table 1 Simulation parameters.

\begin{tabular}{|c|c|c|c|c|c|}
\hline \multirow[t]{4}{*}{$\mathrm{Tx}(\mathrm{CN})$} & $m$ & 1 & Room & Dimensions & $(5 \times 5 \times 3) \mathrm{m}^{3}$ \\
\hline & $\lambda_{0}$ & $850 \mathrm{~nm}$ & & Walls reflectivity & 0.83 (Plaster) \\
\hline & $\Delta \lambda$ & $30 \mathrm{~nm}$ & & Floor reflectivity & 0.87 (Pinewood) \\
\hline & OOK, $\alpha$ & 0.2 & & Ceiling reflectivity & 0.83 (Plaster) \\
\hline \multirow[t]{4}{*}{$\mathrm{Rx}(\mathrm{AP})$} & FOV & $60 \mathrm{deg}$ & Body & Dimensions & $(1.7 \times 0.3 \times 0.2) \mathrm{m}^{3}$ \\
\hline & PD active area & $1 \mathrm{~cm}^{2}$ & & Reflectivity & 0 (Absorbing) \\
\hline & $\mathcal{R}$ & $0.65 \mathrm{~A} / \mathrm{W}$ & & & \\
\hline & TIA $R_{L}$ & $50 \Omega$ & & & \\
\hline
\end{tabular}


For the case of multiple APs, two approaches of equal-gain combining (EGC) and selection combining (SC) are considered. For the former approach, signals received on the APs are simply added together (assuming perfect time synchronization between them) before proceeding to demodulation, whereas the latter approach consists in performing demodulation on the strongest received signal. ${ }^{47}$ To avoid any confusion, it is worth mentioning that we do not consider a multicell architecture here, and the use of multiple APs is only for improving the link reliability.

\section{Channel Characterization}

\subsection{Single AP Configurations}

First, consider the case of a single AP at the center of the ceiling or on the wall, i.e., AP5 or AP8 in Fig. 1(b). Figure 2 shows the time evolution of $H_{0}$, for the two cases of CN1 and CN2 considering the first simulated walk trajectory (i.e., $N_{\text {config }}=516$ ). The abscissa represents the simulation time, with a time step of $\Delta t=0.17 \mathrm{~s}$.

Notice first the larger $H_{0}$ in average for $\mathrm{CN} 1$, compared to $\mathrm{CN} 2$. Interestingly, for a given $\mathrm{CN}$ position, the average $H_{0}$ is more or less the same, regardless of the position of the AP. The mean $H_{0}$ corresponding to $\mathrm{CN} 1$ and $\mathrm{CN} 2$ cases is around -53 and $-55 \mathrm{~dB}$, respectively. As for $H_{0}$ variations, we notice for both AP5 and AP8, larger variations and longer mean time between fades (MTBFs) for CN1 case. For example, the range of $H_{0}$ variations for AP5 is around 9.2 and $4.4 \mathrm{~dB}$, for $\mathrm{CN} 1$ and $\mathrm{CN} 2$ cases, respectively. The corresponding standard deviation is about $3 \mathrm{~dB}$ for $\mathrm{CN} 1$, and it ranges between 1 to $2 \mathrm{~dB}$ for CN2. In addition, compared to AP5, larger $H_{0}$ variations are observed for AP8, with ranges about 12.8 and $7.3 \mathrm{~dB}$, for $\mathrm{CN} 1$ and CN2 cases, respectively. These relatively large variations can be explained by the fact that, compared to AP5, AP8 is more sensitive to body shadowing caused by body rotations during the walk trajectory.

Note that the effects of mobility can be divided into two parts: effects of local mobility resulting from possible shadowing and blockage due to the periodic movements of body parts (legs and arms) and effects of global mobility resulting from the movements of the user inside the room. We can see the effects of local and global mobility in Fig. 2: For the case of CN1, placed on the shoulder and directed toward the ceiling, the link is less subject to shadowing from the body parts. Hence, the observed $H_{0}$ variations are mainly due to global mobility. Depending on the position and the orientation of the user relative to the AP, we may observe a strong or a weak $H_{0}$, with a high range of variations. Also, the absence of periodic patterns in the time evolution of $H_{0}$ for this case supports the argument of negligible local mobility effect on CN1.
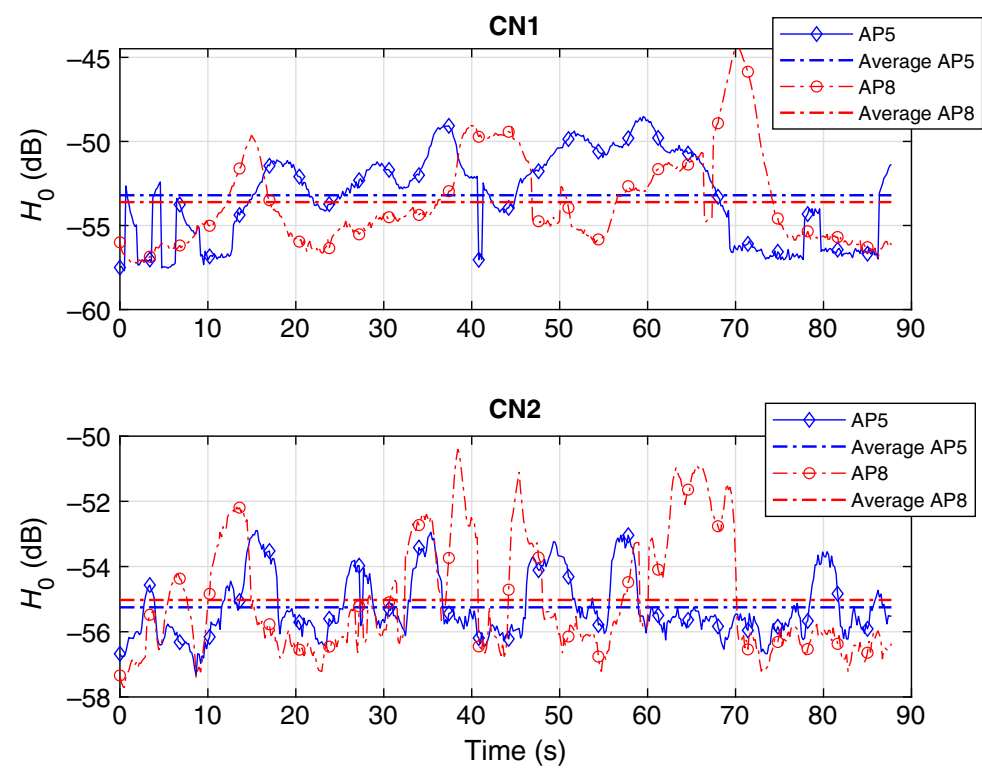

Fig. $2 H_{0}$ variations for the cases of CN1 and CN2 for AP5 and AP8 configurations. 
On the other hand, for the case of $\mathrm{CN} 2$, placed on the waist, the link is more affected by local mobility. For instance, the time evolution of $H_{0}$ is periodic for both links to AP5 and AP8, with different magnitudes for each walk cycle. The latter is attributed to global mobility, whereas the former can be attributed to the shadowing resulting from the periodic swing of the body parts during a walk cycle. For instance, the right arm swinging back and forth might periodically obstruct the link to $\mathrm{CN} 2$.

It is worth mentioning that the observed values of $H_{0}$ are not affected by the user walking speed as far as the walk pattern (i.e., the local mobility model) remains unchanged. This is because the effect of Doppler spread is negligible in OWC networks. ${ }^{12}$ Meanwhile, an increased walking speed results in a slight decrease in the channel coherence time.

The above discussions testify to the significant impact of user mobility on the extra-WBAN optical channel. To reduce this impact, we investigate the use of spatial diversity in the following subsection.

\subsection{Multiple AP Configurations}

Consider the case of using four APs for two configurations of APs placed on the ceiling (i.e., AP1 to AP4) or on the walls (i.e., AP6 to AP9), see Fig. 1(b). Let us first consider the case of individual APs separately. In Fig. 3 the time evolution of $H_{0}$ is shown for the cases of CN1 and $\mathrm{CN} 2$. Note that $\mathrm{CN} 1$ on average has a slightly larger $H_{0}$ for the case of APs located on the ceiling (around $0.5 \mathrm{~dB}$ difference), whereas $\mathrm{CN} 2$ has a larger $H_{0}$ for the case of APs located on the walls: the mean $H_{0}$ for the case of $\mathrm{CN} 1$ and $\mathrm{CN} 2$ is -54 and $-55 \mathrm{~dB}$, respectively. Notice also higher $H_{0}$ variations for the case of $\mathrm{CN} 1$ with the APs located on the ceiling, compared with APs on the walls: the corresponding standard deviation of $H_{0}$ is about 3 and $2 \mathrm{~dB}$, respectively. This can be explained by the fact that the links from CN1 to AP1 to AP4 experience frequent transitions between the availability or absence of the LOS path. On the other hand, CN2 has similar $H_{0}$ variations for both configurations, where its standard deviations are around $1.7 \mathrm{~dB}$.

Interestingly, in the case of $\mathrm{CN} 2$, less correlation is observed between the signals received on different APs. In other words, a higher effective diversity is obtained in this case. This can be explained by the short distance between $\mathrm{CN} 1$ and the Aps, which gives a strong LOS, and the coverage area of CN1 is smaller than $\mathrm{CN} 2$, which makes it subject highly to user's movements.

Figure 4 shows the variations of $H_{0}$ for the cases of EGC and $\mathrm{SC}$ techniques. As expected, the use of multiple APs, in particular with EGC, increases the mean $H_{0}$ and especially reduces its standard deviation due to spatial diversity. Compared to the single AP configuration, the increase in the mean $H_{0}$ is around 5.8 to $6.2 \mathrm{~dB}$ with EGC, and around 2.2 to $2.8 \mathrm{~dB}$ with $\mathrm{SC}$, for both (AP1 to AP4) and (AP6 to AP9) cases. Meanwhile, compared with CN2, we notice a higher average $H_{0}$ (about 1.1 to $2.6 \mathrm{~dB}$ ) and larger $H_{0}$ variations (about 1.4 to $3.6 \mathrm{~dB}$ ) for the case of
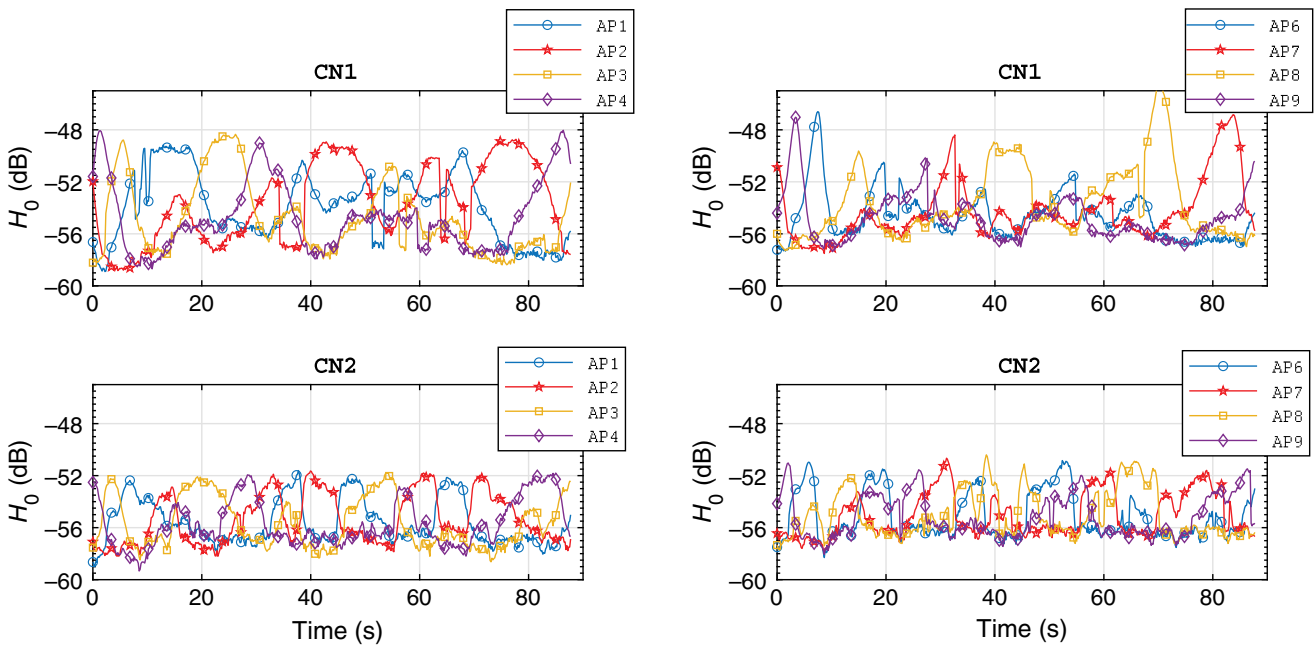

Fig. $3 H_{0}$ variations for the cases of $\mathrm{CN} 1$ and $\mathrm{CN} 2$ considering different APs on the ceiling (AP1 to AP4) and on the walls (AP6 to AP9). 

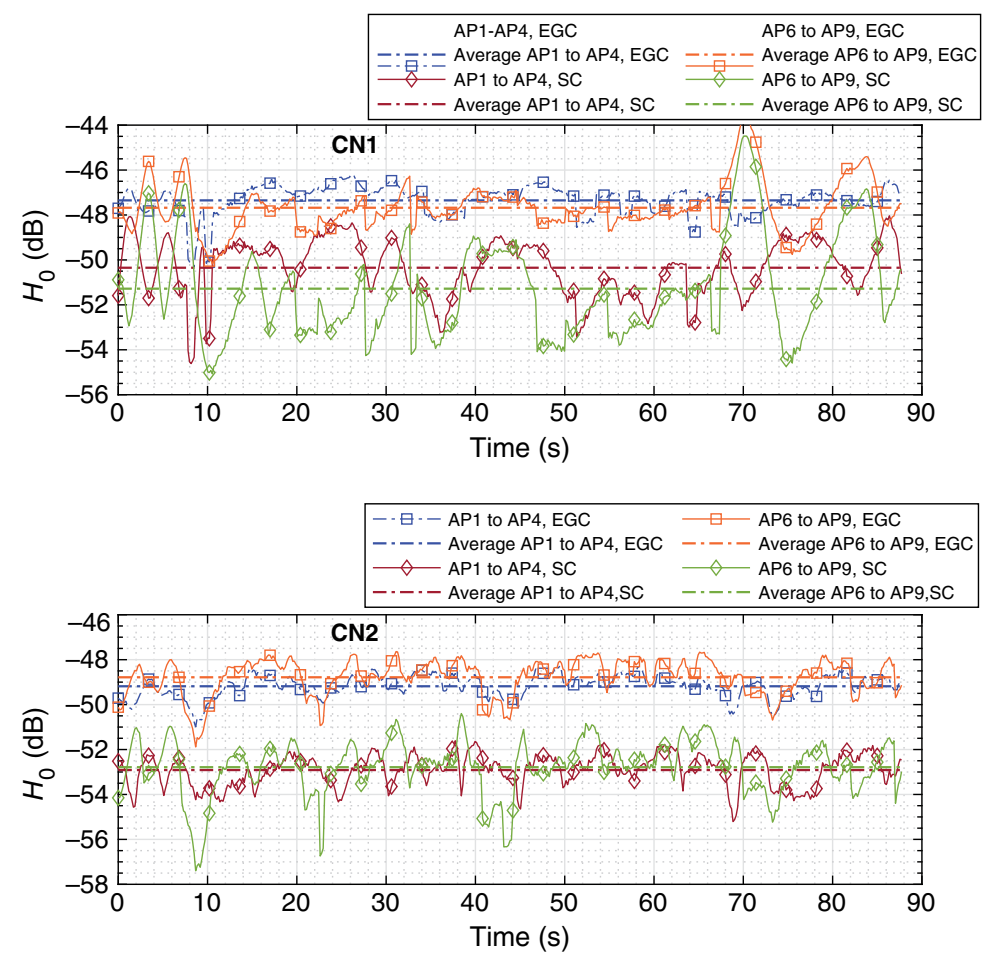

Fig. $4 H_{0}$ variations for the cases of $\mathrm{CN} 1$ and $\mathrm{CN} 2$ for the multiple-AP configurations using EGC and SC detection methods.

$\mathrm{CN} 1$, similar to the single AP configurations. Also, larger variations and shorter MTBFs are observed for the case of (AP6 to AP9), compared with (AP1 to AP4). This would suggest that the (AP1 to AP4) configuration is more robust against user mobility effects. Based on this, in the sequel, only the case of (AP1 to AP4) configuration is considered.

\section{Link Performance Analysis}

\subsection{Statistical Model of Channel Gain}

As explained in Sec. 2.3, to calculate the link BER, $H_{0}$ is generated randomly based on Gaussian-kernel KDE for the considered scenarios. For this, we reasonably combine the data of the two generated trajectories to increase the accuracy of the statistical estimation and also to have a better representation of the movement area. For instance, in Fig. 5, the 1103 samples histograms of the simulated $H_{0}$ for the case of $\mathrm{CN} 1$ via Opticstudio are presented together with the KDE-based PDF fits. The corresponding KDE parameters are specified in Table 2.

First, notice a good fit between the histograms of the simulated data and the estimated PDFs, in particular for the multiple AP cases with EGC. Also, the reduction in the histogram spread (in particular, for EGC) shows the benefit of spatial diversity in reducing shadowing and link blockage effects.

\subsection{Performance Comparison of Single and Multiple AP Configurations}

Using the obtained approximate PDFs, we randomly generate more than $10^{7}$ channel realizations. For each generated $H_{0}$, the BER is calculated from Eq. (6). Given the slow time-varying nature of the channel, the outage probability $P_{\text {out }}$ is considered for the link performance analysis, which is defined as the probability that the instantaneous link BER goes more than a given threshold $\mathrm{BER}_{\mathrm{th}}=10^{-3}$. For the case of PIN-based Rx, Fig. 6 shows plots of $P_{\text {out }}$ versus $P_{t}$ for $R_{b}$ ranging between $10 \mathrm{kbps}$ to $1 \mathrm{Mbps}$, for the cases of $\mathrm{CN} 1$ and $\mathrm{CN} 2$, and single or multiple APs on the ceiling, i.e., AP5 or (AP1 to AP4). Considering first the single AP case, notice the degradation of 


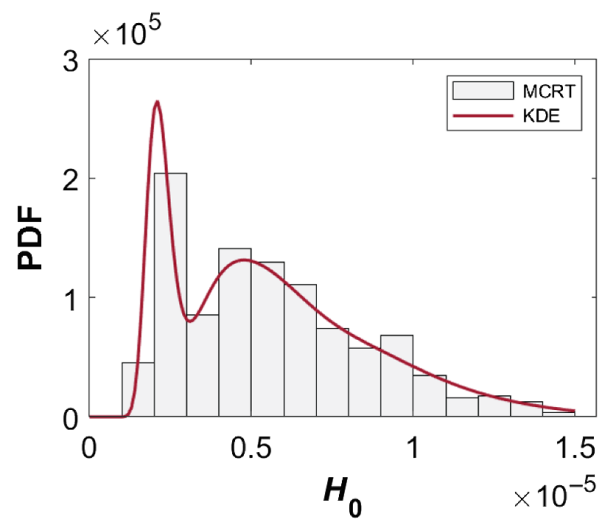

(a)

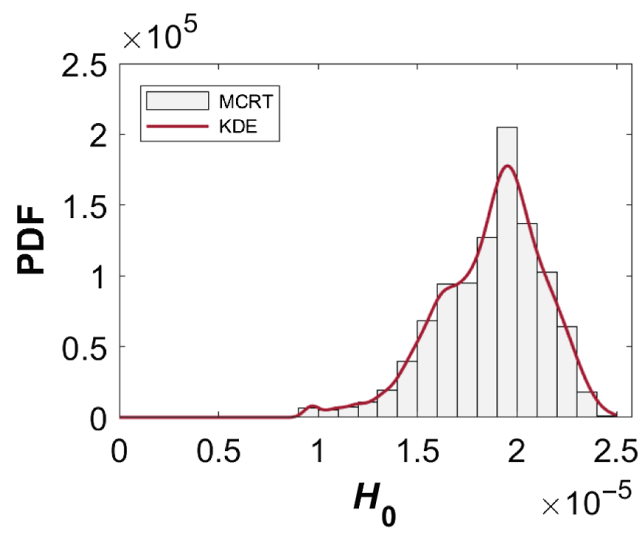

(b)

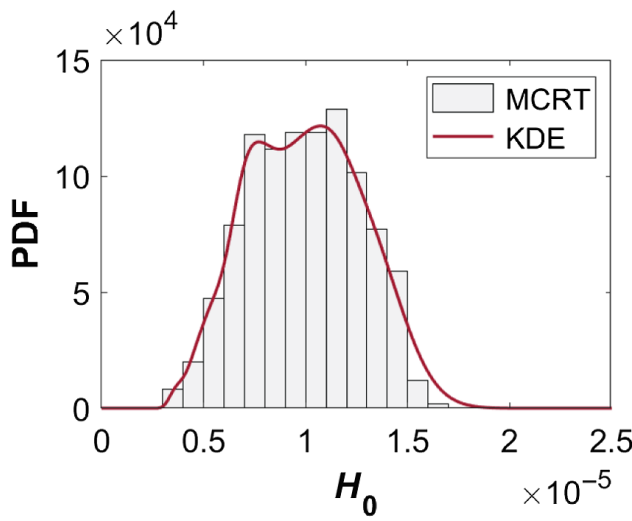

(c)

Fig. 5 Histograms of $H_{0}$ (in linear scale) for 1103 channel realizations and estimated PDF by KDE with Gaussian kernel: (a) CN1 and AP5, (b) CN1 and AP1 to AP4 with EGC, and (c) with SC.

Table 2 Calculated smoothing parameter $\rho$.

\begin{tabular}{lcccc}
\hline \hline Configuration & & & CN1 & CN2 \\
\hline Single AP & \multicolumn{2}{c}{ Ceiling (AP5) } & 0.1579 & 0.0402 \\
& \multicolumn{2}{c}{ Wall (AP8) } & 0.1115 & 0.0731 \\
Multiple AP & Ceiling & EGC & 0.0339 & 0.0314 \\
& & SC & 0.0813 & 0.0462 \\
& Walls & EGC & 0.0453 & 0.0417 \\
& & SC & 0.1138 & 0.0608 \\
\hline \hline
\end{tabular}

$P_{\text {out }}$ with an increase in $R_{b}$, as expected. In fact, the Rx LPF bandwidth is changed according to $R_{b}$. Consequently, for increased $R_{b}$, the Rx noise variance increases, resulting in a BER degradation. For the multiple AP case, from Figs. 6(c) and 6(d), for a given $R_{b}$, we reasonably note a considerably lower requirement of $P_{t}$ with EGC to attain a target $P_{\text {out }}$. For instance, for $P_{\text {out }}=$ $10^{-3}$ and $R_{b}=100 \mathrm{kbps}$, the required $P_{t}$ is around 5.5 and $18 \mathrm{~mW}$ for the cases of EGC and SC signal detection, respectively, compared with $40.5 \mathrm{~mW}$ for the case of single AP from Fig. 6(a). This elucidates the tangible enhancement of the link reliability achieved by spatial diversity. Another interesting result is that the $\mathrm{CN} 2$ case offers a better performance than $\mathrm{CN} 1$ for the single AP configuration. This can be explained by the fact that the link to $\mathrm{CN} 1$ depends highly on the availability of the LOS, whereas the link using $\mathrm{CN} 2$ depends rather on first-order reflections. 


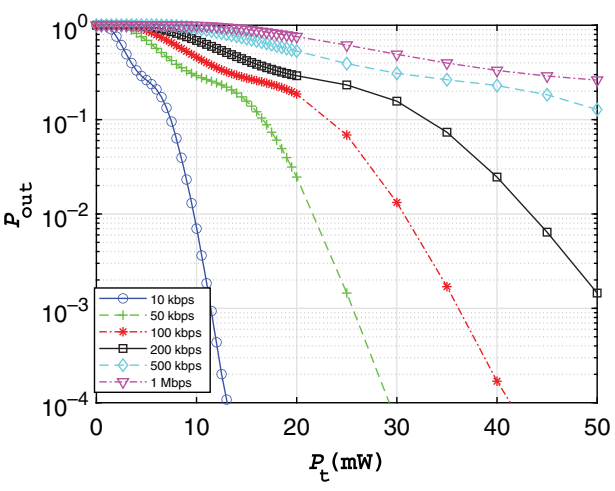

(a)

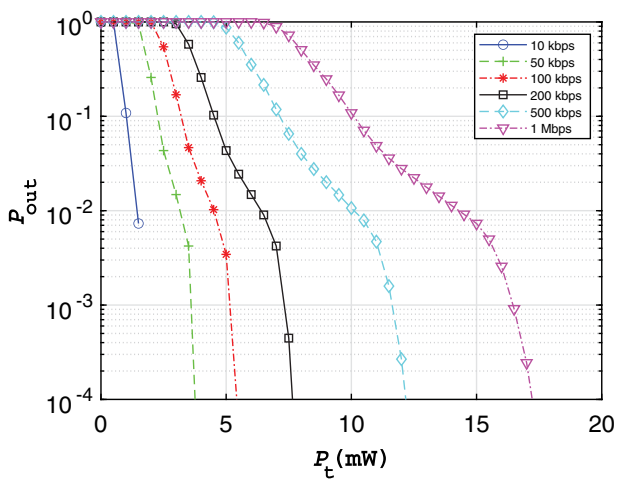

(c)

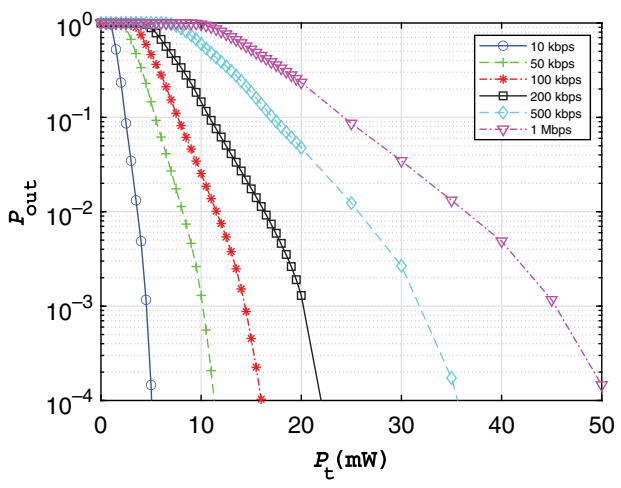

(e)

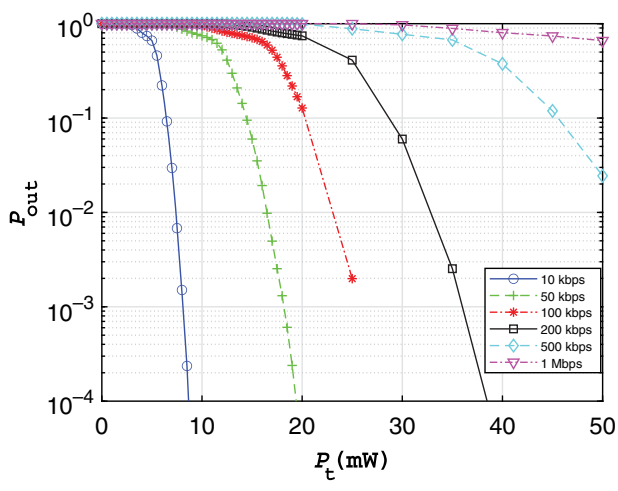

(b)

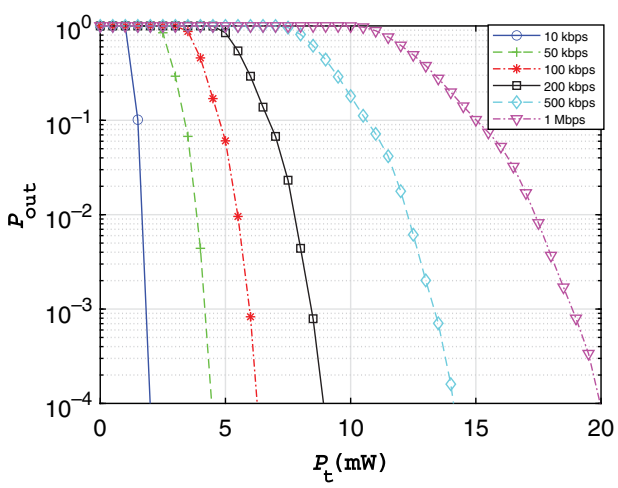

(d)

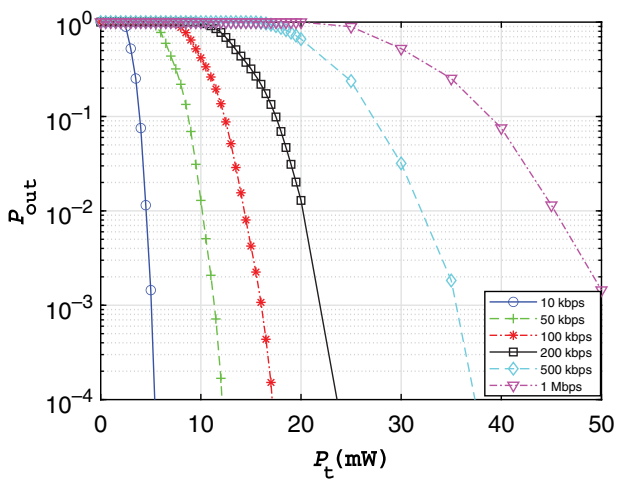

(f)

Fig. $6 P_{\text {out }}$ versus the transmit power for different data rates for the cases of (a) CN1 and AP5, (b) CN2 and AP5, (c) CN1 and AP1 to AP4 with EGC, (d) CN2 and AP1 to AP4 with EGC, (e) CN1 and AP1 to AP4 with SC, and (f) CN2 and AP1 to AP4 with SC. Uncoded OOK modulation.

Given that the availability of LOS is less likely than that of first- and higher-order reflections, the link to $\mathrm{CN} 2$ can be considered to be more stable. However, for the multiple AP configuration, the links to CN1 and CN2 have similar performance for both EGC and SC strategies. This similarity is again due to the spatial diversity that increases the likelihood of LOS and diffuse link availability, with the result of reduced shadowing and link blockage effect.

\subsection{Performance Comparison of PIN- and APD-based Rxs}

Figure 7 shows the performance of PIN and APD versus $I_{b}$ with the two combination strategies for the case of CN1 and CN2, $P_{t}=15 \mathrm{~mW}$ and $R_{b}=200 \mathrm{kbps}$ (recall that $I_{b}$ denotes the average photocurrent due to background noise). 


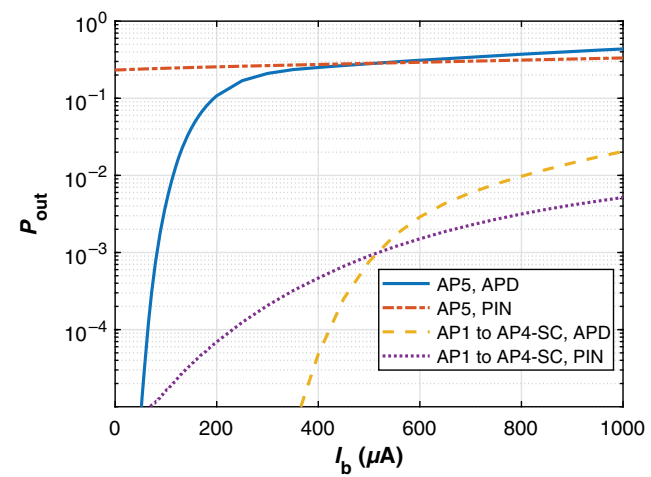

(a)

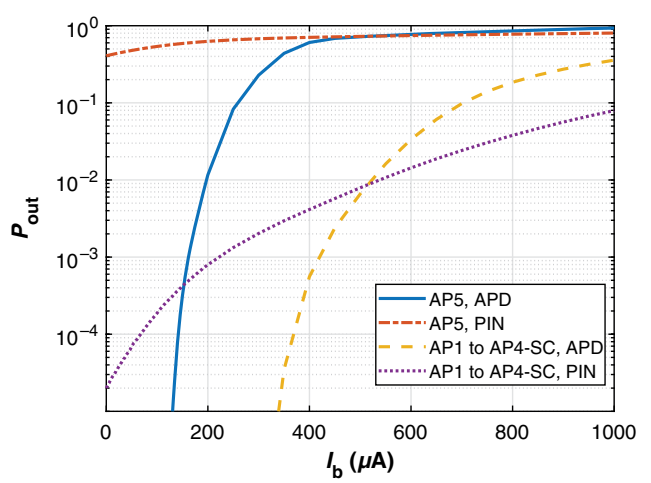

(b)

Fig. $7 P_{\text {out }}$ versus background noise current $I_{b}$ for PIN- and APD-based Rxs with different AP configurations: (a) CN1, (b) CN2. Uncoded OOK modulation. $P_{t}=15 \mathrm{~mW}, R_{b}=200 \mathrm{kbps}$.

First, as it could be expected, the multiple AP configuration with EGC offers the best performance for both cases of $\mathrm{CN} 1$ and $\mathrm{CN} 2$. The corresponding $P_{\text {out }}$ is too low for the considered $I_{b}$, this is why they are not shown in the figure. From Fig. 7(a), considering a target $P_{\text {out }}=10^{-3}$ and the APD-based Rx, the link can operate up to an $I_{b}$ of 80 and $510 \mu \mathrm{A}$, for the cases of AP5 and AP1 to AP4 with SC detection, respectively. Also, as was expected ${ }^{35}$ the performance of the APD-based Rx is more affected by $I_{b}$, compared to the PIN-based Rx. For example, from Fig. 7(b), considering AP1 to AP4 with SC detection, for $I_{b}$ up to $525 \mu \mathrm{A}$, the APD-based $\mathrm{Rx}$ has a better performance, whereas the inverse holds for $I_{b}>525 \mu \mathrm{A}$. Interestingly, the case of single AP with APD-based Rx outperforms the PIN-based multiple AP with SC detection for $I_{b} \lesssim 155 \mu \mathrm{A}$. This $I_{b}$ corresponds to environments with filtered ambient indirect sunlight. ${ }^{36,48}$

\section{Conclusions}

This work investigated channel characterization and performance study for medical optical extra-WBAN links, taking into account user mobility using a dynamic model based on a 3D animation of the user walk cycle and a modified RWP model. Based on an accurate MCRT approach, we calculated the CIR and the channel DC gain and studied the impact of the $\mathrm{CN}$ position on the user body and AP placement at different locations of the room. The presented results and analysis are quite insightful in the design of medical WBANs by taking into account practical and realistic system parameters and channel characteristics.

In particular, we demonstrated the benefit of using multiple-APs with EGC and SC combining strategies in improving link reliability due to the reduction of beam shadowing during user movements, which justifies the increased network implementation complexity. Placing the APs on the ceiling was found to provide a better performance, given the lower DC gain variations it offers. Also, our study revealed that the placement of the $\mathrm{CN}$ on the waist is more advantageous, as compared to the position on the shoulder, especially for the single AP configuration. Finally, we investigated the use of PIN- and APD-based Rxs and showed that except under high background noise conditions, using one APD-based AP provides acceptable link performance. However, when working under moderate-to-strong background noise conditions, we recommend using multiple PIN-based APs.

Future work can consider the case of multiple users in a room and the development of efficient multiple-access techniques, as in Ref. 21. Also, to use in large indoor spaces, appropriate multicell architectures need to be designed with the possibility of hand-over, supporting user mobility.

\section{Acknowledgments}

This work has been supported by VisIoN, a European project funded by the European Union's Horizon 2020 research and innovation program under the Marie Skłodowska-Curie Grant 
Agreement No. 764461. This paper is also based upon work from COST Action CA19111 (European Network on Future Generation Optical Wireless Communication Technologies, NEWFOCUS), supported by COST (European Cooperation in Science and Technology). Parts of this work were presented at the SPIE 2020 Photonics West Conference, San Francisco, CA (Ref. 27). The authors declare no conflicts of interest.

\section{References}

1. T. Botsis and G. Hartvigsen, "Current status and future perspectives in telecare for elderly people suffering from chronic diseases," J. Telemed. Telecare 14, 195-203 (2008).

2. J. Kim et al., "A $112 \mathrm{~Gb} / \mathrm{s}$ PAM-4 transmitter with 3-tap FFE in $10 \mathrm{~nm}$ CMOS," in IEEE ISSCC Conference, San Francisco, pp. 102-104 (2018).

3. P. Abiri et al., "Inductively powered wireless pacing via a miniature pacemaker and remote stimulation control system," Sci. Rep. 7, 6180 (2017).

4. L. Atzori, A. Iera, and G. Morabito, "The internet of things: a survey," Comput. Netw. 54(15), 2787-2805 (2010).

5. "IEEE standard for local and metropolitan area networks-Part 15.6: wireless body area networks," in IEEE Std 802.15.6-2012, pp. 1-271 (29 February 2012).

6. H. Ren and M. Q. H. Meng, "Understanding the mobility model of wireless body sensor networks," in Int. Conf. Inf. Acquisition, Weihai, China, pp. 306-310 (2006).

7. M. Nabi, M. Geilen, and T. Basten, "MoBAN: a configurable mobility model for wireless body area networks," in Int. ICST Conf. Simul. Tools and Tech., Barcelona, Spain, pp. 168-177 (2011).

8. S. L. Cotton, W. G. Scanlon, and B. K. Madahar, "Millimeter-wave soldier-to-soldier communications for covert battlefield operations," IEEE Commun. Mag. 47, 72-81 (2009).

9. M. Mackowiak and L. M. Correia, "A statistical model for the influence of body dynamics on the gain pattern of wearable antennas in off-body radio channels," Springer Wireless Personal Commun. 73, 381-399 (2013).

10. K. Turbic, L. M. Correia, and M. Beko, "A mobility model for wearable antennas on dynamic users," IEEE Access 6, 63635-63648 (2018).

11. O. Haddad and M. A. Khalighi, "Enabling communication technologies for medical wireless body-area networks," in Global LiFi Congr., Paris, France, pp. 1-5 (2019).

12. Z. Ghassemlooy et al., Visible Light Communications: Theory and Applications, CRC Press, Boca Raton, Florida (2017).

13. A. Julien-Vergonjanne, S. Sahuguède, and L. Chevalier, "Optical wireless communications: an emerging technology," in Optical Wireless Body Area Networks for Healthcare Applications, pp. 569-587, Springer, Cham, Switzerland (2016).

14. O. Haddad et al., "Channel characterization and modeling for optical wireless body-area networks," IEEE Open J. Commun. Soc. 1, 760-776 (2020).

15. O. Haddad et al., "Performance analysis of optical intra-WBAN links," in Int. Symp. Commun. Syst., Networks and Digital Signal Process., Porto, Portugal, pp. 1-6 (2020).

16. C. Le Bas, S. Sahuguède, and A. Julien-Vergonjanne, "Theoretical and experimental approach for the design of an optical wireless physical activity monitoring system," Int. J. Wirel. Inf. Netw. 24, 65-77 (2017).

17. A. Behlouli et al., "Impact of physical and geometrical parameters on visible light communication links," in Adv. Wireless and Optical Commun., Riga, Latvia, pp. 73-76 (2017).

18. D. R. Dhatchayeny, S. Arya, and Y. H. Chung, "Patient mobility support for indoor nondirected optical body area networks," Sensors 19(10), 2297 (2019).

19. T. B. Hoang, S. Sahuguède, and A. Julien-Vergonjanne, "Optical wireless network design for off-body-sensor based monitoring," Wireless Commun. Mob. Comput. 2019, 1-13 (2019).

20. B. Donmez, R. Mitra, and F. Miramirkhani, "Channel modeling and characterization for VLC-based medical body sensor networks: trends and challenges," IEEE Access $\mathbf{9}$, 153401-153419 (2021).

21. M. J. Hasan et al., "Performance analysis of optical-CDMA for uplink transmission in medical extra-WBANs," IEEE Access 8, 171672-171685 (2020). 
22. M. J. Hasan, M. A. Khalighi, and B. Béchadergue, "Experimental implementation of optical-CDMA for medical extra-WBAN links," in Int. Symp. Commun. Syst., Networks and Digital Signal Process., Porto, Portugal, pp. 1-6 (2020).

23. "Opticstudio," https://www.zemax.com/products/opticstudio (accessed 16 September 2019).

24. H. B. Eldeeb et al., "Channel measurements and ray tracing simulations for MIMO light communication at $200 \mathrm{MHz}$," in IEEE Photonics Conf., Vancouver, Canada, pp. 1-2 (2020).

25. J. R. Barry et al., "Simulation of multipath impulse response for indoor wireless optical channels," IEEE J. Sel. Areas Commun. 11, 367-379 (1993).

26. D. B. Smith and L. W. Hanlen, "Ultra-low-power short-range radios," in Channel Modeling for Wireless Body Area Networks, pp. 25-55, Springer International Publishing, Cham, Switzerland (2015).

27. O. Haddad, M. A. Khalighi, and S. Zvanovec, "Channel characterization for optical extraWBAN links considering local and global user mobility," Proc. SPIE 11307, 113070G (2020).

28. "3D mesh,” https://3docean.net/item/low-poly-base-mesh-male/73426?WT.ac=category_ thumbWT.seg_1=category_thumb WT.z_author=karan8/ (accessed 27 June 2020).

29. "Blender," https://www.blender.org/ (accessed 23 September 2019).

30. "Animation tutorial," https://www.blendernation.com/2020/05/16/rig-anything-with-rigify3-prebuilt-meta-rigs-biped-quadruped/ (accessed 27 June 2020).

31. C. Bettstetter, H. Hartenstein, and X. Pérez-Costa, "Stochastic properties of the random waypoint mobility model," Wirel. Netw. 10, 555-567 (2004).

32. S. McKinley and M. Levine, "Cubic spline interpolation," College Redwoods 45, 1049-1060 (1998).

33. J. E. Graham et al., "Walking speed threshold for classifying walking independence in hospitalized older adults," Phys. Ther. 90, 1591-1597 (2010).

34. R. P. Duncan, M. E. McNeely, and G. M. Earhart, "Maximum step length test performance in people with Parkinson disease: a cross-sectional study," J. Neurol. Phys. Therapy 41, 215-221 (2017).

35. F. Xu, M. Khalighi, and S. Bourennane, "Impact of different noise sources on the performance of PIN- and APD-based FSO receivers," in Int. Conf. Telecommun., Graz, Austria, pp. 211-218 (2011).

36. F. R. Gfeller and U. Bapst, "Wireless in-house data communication via diffuse infrared radiation," Proc. IEEE 67, 1474-1486 (1979).

37. D. W. Scott, "Kernel density estimators," in Multivariate Density Estimation, pp. 125-193, John Wiley \& Sons Ltd., New York (1992).

38. E. L. Lehmann, "Model specification: the views of Fisher and Neyman, and later developments," Stat. Sci. 5(2), 160-168 (1990).

39. A. Z. Zambom and R. Dias, "A review of kernel density estimation with applications to econometrics," Int. Econometric Rev. 5, 20-42 (2013).

40. V. Epanechnikov, "Non-parametric estimation of a multivariate probability density," Theory Prob. Appl. 14, 153-158 (1969).

41. B. Silverman, Density Estimation for Statistics and Data Analysis, Chapman \& Hall/CRC, London (1986).

42. M. T. Dabiri, S. M. S. Sadough, and M. A. Khalighi, "FSO channel estimation for OOK modulation with APD receiver over atmospheric turbulence and pointing errors," Opt. Commun. 402, 577-584 (2017).

43. F. Miramirkhani and M. Uysal, "Channel modeling and characterization for visible light communications," IEEE Photonics J. 7, 1-16 (2015).

44. L. Chevalier, S. Sahuguède, and A. Julien-Vergonjanne, "Optical wireless links as an alternative to radio-frequency for medical body area networks," IEEE J. Sel. Areas Commun. 33, 2002-2010 (2015).

45. T. Haran and S. Chien, "Infrared reflectivity of pedestrian mannequin for autonomous emergency braking testing," in IEEE Int. Conf. Intell. Transp. Syst., Rio de Janeiro, Brazil, pp. 2230-2235 (2016).

46. S. Movassaghi et al., "Wireless body area networks: a survey," IEEE Commun. Surv. Tutorials 16, 1658-1686 (2014). 
47. J. G. Proakis and M. Salehi, Digital Communications, 5th ed., McGraw-Hill, New York (2007).

48. M. A. Khalighi et al., "Double-laser differential signaling for reducing the effect of background radiation in free-space optical systems," IEEE/OSA J. Opt. Commun. Networking 3, 145-154 (2011).

Oussama Haddad received his BSc and MSc degrees in electrical engineering from National Polytechnic School of Algiers, Algeria, in 2017, and his PhD from Fresnel Institute, École Centrale Marseille, France, in 2021. He is currently working as a research and teaching assistant at the Microelectronics and Telecommunications Department of Polytech-Marseille, France. His research interests include optical wireless communications and wireless body area networks.

Mohammad-Ali Khalighi is an associate professor at École Centrale Marseille, Marseille, France, and head of the Optical Communications for IoT group at Fresnel Institute Research Lab. He is currently serving as project coordinator for the H2020 ITN MSCA VisIoN project (visible-light-based interoperability and networking) and chair of the COST Action CA19111 NEWFOCUS (European Network on Future Generation Optical Wireless Communication Technologies). He is also serving as editor-at-large for the IEEE Transactions on Communications and has served as an associate editor for the IET Electronics Letters as well as guest editor for the IEEE Open Journal of the Communications Society and Elsevier Optik Journal. His main research interests include signal processing for wireless communication systems with an emphasis on free-space, underwater, and indoor visible-light optical communications. He is a senior member of IEEE.

Stanislav Zvanovec received his MSc and $\mathrm{PhD}$ degrees from Czech Technical University in Prague in 2002 and 2006, respectively, where he is a full professor, the deputy head of the Department of Electromagnetic Field, and a leader of the Wireless and Fiber Optics Team. He has authored two books and more than 250 journal articles and conference papers. His current research interests include FSO and fiber optical systems, VLC, and RF over optics. He is a senior member of IEEE. 